\title{
Efecto de un programa de educación física con intensidad moderada vigorosa sobre el desarrollo motor en niños de preescolar \\ Effect of a physical education program with moderate-to-vigorous intensity on motor development in preschool children \\ *Daniel Alejandro Piña Díaz, *Paulina Yesica Ochoa-Martínez, *Javier Arturo Hall-López, *Zeltier Edier Reyes Castro, **Edgar Ismael Alarcón Meza, **Luis Roberto Monreal Ortiz, ***Pedro Sáenz-López Buñuel \\ *Universidad Autónoma de Baja California (México), **Universidad Autónoma de Sinaloa (México), ***Universidad de Huelva
} (España)

Resumen. Objetivo: Efecto de un programa de educación física con intensidad moderada vigorosa sobre el desarrollo motor en niños de preescolar. Método: Participaron 20 alumnos de tercer grado de preescolar, 13 mujeres y 7 hombres, con una edad de $5.05 \pm 0.2$ años, en un programa de educación física con intensidad moderada a vigorosa adaptado al modelo pedagógico CATCH (avance coordinado para la salud de los niños por sus siglas en inglés), las sesiones se llevaron a cabo 3 veces por semana, durante 12 semanas. La intensidad de la actividad física se determinó mediante el sistema para observar el tiempo de instrucción en la actividad física (SOFIT), antes y después del programa el desarrollo motor fue evaluado mediante el inventario de desarrollo Battelle valorando las variables de coordinación corporal, locomoción, motricidad fina y habilidad perceptiva, que determinan la puntuación de motricidad gruesa y fina. Resultados: La intensidad promedio de la actividad física moderada a vigorosa fue $65 \%$ del tiempo total de la clase de educación física, el análisis estadístico mediante el test t-Student para muestras relacionadas, reportó diferencias significativas en la puntuación de motricidad gruesa ( $\mathrm{p}=0.00$ ) y motricidad fina $(\mathrm{p}=0.00$ ), antes y después de la intervención, el porcentaje de cambio resultó $25.5 \mathrm{~A} \%$ y $11.3 \ddot{\mathrm{A}} \%$ respectivamente. Conclusión: a pesar que el programa CATCH reporta modestos resultados en variables asociadas a la obesidad en preescolares, su aplicación durante tres meses mostro una influencia positiva para la mejora del desarrollo motor en niños de edad preescolar.

Palabras clave: Educación Física, Escuela, Desarrollo Motor.

\begin{abstract}
Objective: To evaluate the effect of a physical education program with moderate-to-vigorous intensity on motor development in kindergarten students. Method: a total of 20 preschool students with average age of $5.05 \pm 0.2$ years old were involved ( 13 girls and 7 boys); they carried out a program of physical education at moderate-to-vigorous intensity, based on the pedagogical model of CATCH (Coordinated Approach to Child Health). The program was performed during 3 months, with 3 sessions per week. Intensity of physical activity was determined by the system for observing fitness instruction time (SOFIT), Motor development was determined using the education Battelle developmental inventory, assessing the variables of body coordination, locomotion, fine motor and perceptual ability, which determine the gross and fine motor score. Results: The average engagement time at moderate-to-vigorous physical activity intensity was $65 \%$ of the total time of the physical education class. The Student's t-test for related samples was run for the analysis. It reported significant differences in both gross $(\mathrm{p}=0.00)$ and fine motor scores $(\mathrm{p}=0.00)$, before and after intervention; the percentage of change was $25.5 \ddot{\mathrm{A}} \%$ and $11.3 \ddot{\mathrm{A}} \%$, respectively. Conclusion: Although the CATCH program reports modest results in variables associated with obesity in preschoolers, its application for three months showed positive influence for the improvement of motor development in preschoolers.
\end{abstract}

Keywords: Physical Education, School, Motor Development.

\section{Introducción}

La Organización Mundial de la Salud (OMS), menciona que el desarrollo en la primera infancia (etapa prenatal hasta los ocho años de edad), es crucial para su optimo bien estar, crecimiento y es una influencia clave en el posterior ciclo de vida de un individuo (OMS, 2016); investigaciones han demostrado de manera significativa, que el desarrollo motor es mejor en estudiantes de preescolar que cuentan con profesor de educación física (Luna, y Poblete, 2011, Luarte, Poblete, y Flores, 2014, Bermúdez, y col., 2018), un estudio ha identificado, que entre más tiempo dedicado a clases de educación física por estudiantes de preescolar su desarrollo motor es más favorecido (Jiménez Díaz y Araya Vargas, 2010), otra investigación evalúo la variable de edad motora equivalente en niños de preescolar, utilizando como instrumento el inventario de desarrollo y correlacionó que los niños con una adquisición motora esperada para su edad presentan, en mayor medida un igual desarrollo cognitivo y lenguaje acor-

Fecha recepción: 30-07-19. Fecha de aceptación: 25-12-19 Paulina Yesica Ochoa-Martínez

pochoa@uabc.edu.mx de a su edad (Campo Ternera, 2010). La Organización Mundial de la Salud (OMS) recomienda que los niños entre 5 a 17 años de edad dediquen como mínimo 60 minutos diarios en actividad física con intensidad moderada a vigorosa (OMS, 2014). Revisiones sistemáticas y meta-análisis mencionan que niños sedentarios presentan en mayor medida riesgo a presentar patologías asociadas a la obesidad (Brooke y col., 2014; Sims, Scarborough, y Foster, 2015). Respecto a este factor una investigación reporta que el $16.4 \%$ de los preescolares presenta un nivel de actividad física activa y muy activa (Mollinedo Montaño y col., 2012), esto se asocia con otra investigación en preescolares reportando que permanece un $69 \%$ del tiempo en actividades sedentarias durante la jornada escolar (Schlechter, y col., 2017). Existe evidencia que apoya la relación entre la obesidad y sedentarismo infantil y un bajo rendimiento en pruebas psicomotoras (Cigarroa, Sarqui, y Zapata-Lamana, 2016). A pesar que la obesidad es un problema multifactorial y complejo en su etiología se ha identificado a la educación básica como área de oportunidad para promover un estilo de vida saludable y activo desde edades tempranas (Kim, 2012, Cuadri Fernández y col., 2017, debido a su amplia cobertura es un contexto ideal para la promoción y desarrollo de actitudes positivas y 
hábitos saludables, que sirvan de base para que los niños tiendan a futuro adoptar un estilo de vida activo como adultos (Langford y col., 2015; Lonsdale y col., 2013) y de esta manera mejorar la calidad de vida y reducir los costos en el tratamiento relacionados al sedentarismo y la obesidad (Kim, 2012). Por lo que el profesorado de educación física juega un papel importante en la conducción de contenidos en los programas que favorecen por medio del movimiento el desarrollo motriz en prescolar dentro de las cuales está la optimización del tiempo en la conducción de la clase de educación fisca (Ruiz Heredia y col., 2018) y actividad física moderada vigorosa a manera de juego (Palma, Pereira, y Valentini, 2014, Gil-Espinosa, Romance García, y Nielsen Rodríguez, (2018). Para disminuir esta problemática, se han realizado múltiples programas de intervención e escolares, tal es el caso del programa Coordinated Approach to Child Health $(\mathrm{CATCH})$, que ha mostrado modestos resultados en disminuir el sobrepeso y obesidad en preescolares (Shreela y col., 2019), Aun sin embargo, en el contexto de la educación física, ha resultado efectivo para para mejorar la calidad de la clase de educación física en cuanto a incrementar la actividad física moderada a vigorosa e incrementar las oportunidades de participar por los estudiantes (McKenzie, y col., 1994), por lo anterior, el presente estudio tiene como objetivo conocer el efecto de un programa de educación física, utilizando como modelo el programa CATCH con el componente de actividad física moderada vigorosa para valorar el desarrollo motor en niños de preescolar.

\section{Metodología}

\section{Participantes}

El estudio forma parte de una investigación registrada en la Coordinación de posgrado e investigación (CPI UABC) con la clave 149/1823. Se llevó a cabo bajo un diseño metodológico cuasi-experimental con muestreo no probabilístico por conveniencia, donde la variable independiente (programa de educación física en preescolar adaptado al modelo pedagógico $\mathrm{CATCH}$ ) se manipula para medir sus efectos sobre la variable dependiente (desarrollo motor), con el propósito de determinar el grado de cambio producido por el tratamiento, estableciendo una relación causa efecto (Thomas, Nelson y Silverman 2015), siguiendo los principios éticos de investigación en seres humanos de la declaración de Helsinki (Puri y col., 2009).

Se reclutaron 20 alumnos 7 niños y 13 niñas de $5.05 \pm 0.2$ años de edad, de 3er año de preescolar del «Jardín de niños Real del Castillo», cabe resaltar que los participantes previamente no contaban con profesor de educación física, solo realizan la actividad física que les brindan las educadoras acorde al programa educativo para prescolar.

Se realizó una reunión con los padres de familia o tutores, quienes fueron informados, sobre el programa, instrumentos y procedimientos para aplicar en el estudio, así como los riesgos que podrían presentarse y beneficios derivados de este, avalando mediante carta de consentimiento la aceptando para participar.

\section{Instrumentos}

Se utilizó el inventario de desarrollo Battelle, (Newborg,
Stock, y Wnek, 1996). El cual es una batería para evaluar las habilidades fundamentales del desarrollo en niños comprendiendo desde el nacimiento y los ocho años de edad, su aplicación es individual y está tipificada. Es un inventario objetivo que, durante sus procedimientos para la obtención de datos, utiliza la observación y usa un examen estructurado. Los ítems se presentan en un formato normalizado que especifica la conducta a evaluar, los materiales necesarios, procedimientos de administración y criterios para puntuar la respuesta. Su aplicación está compuesta por 341 ítems para el total del rango de edades al que está destinada. Examina las áreas del desarrollo personal/social, adaptativa, motora y comunicación, para los fines de la investigación se determinó el área de motora (82 ítems), que consta de 5 sub áreas.

-Control Muscular: Evalúa el desarrollo motor grueso y la capacidad del niño para establecer y mantener el control, principalmente sobre los músculos que utiliza para sentarse, estar de pie, pasar objetos de una mano a otra y realizar tareas semejantes (en esta investigación esta variable será descartada ya que no concuerda con la edad de la muestra). -Coordinación Corporal: Evalúa aspectos del desarrollo motor grueso: la capacidad del niño para utilizar su sistema muscular y para establecer un control y una coordinación corporal cada vez mayores (por ejemplo, cambiar la posición del cuerpo, rodar en el suelo, dar patadas, tirar y recoger objetos, saltar, hacer flexiones). -Locomoción: Evalúa aspectos del desarrollo motor grueso: La capacidad del niño de utilizar los sistemas de musculatura de forma integrada con el fin de trasladarse de un sitio a otro (por ejemplo, arrastrarse, gatear, andar, correr, saltar o subir y bajar escaleras), que sumados arrojan la puntación total de la Motricidad Gruesa, -Motricidad Fina: Evalúa el desarrollo del control y coordinación muscular del niño, especialmente de la musculatura fina de brazos y manos que permite llevar a cabo tareas cada vez más complejas como tomar y soltar objetos, abrir y cerrar puertas y cajones, ensartar cuentas, pasar páginas, cortar, doblar papel y utilizar el lápiz correctamente. -Motricidad Perceptiva: Evalúa aspectos del desarrollo motor fino: la capacidad del niño para integrar la coordinación muscular y las habilidades perceptivas en actividades concretas, como formar torres, colocar anillas en un soporte, copiar círculos, cuadrados, dibujar y escribir, que sumados arrojan la puntación total de la Motricidad Fina. La prueba se realizó en entre una hora a una hora y treinta minutos. Para registrar los valores en los ítems al evaluar se tomó 0 como nunca, 1 como a veces y 2 siempre, realizando suma que proporciona una puntuación total cuyo resultado se compara con las tabla de resumen de puntuaciones y perfil del inventario, estableciendo así, la edad motora en meses equivalente a partir de los 82 ítems de las 5 sub áreas, clasificándose como: 1.-Alto: Por encima de lo esperado para su edad, 2.-Normal: de acuerdo a los patrones normativos de la edad y 3.-Bajo: por debajo de lo esperado de su edad. En este caso eliminaremos la primera sub área ya que no está acorde para la edad del niño del presente estudio siendo que solo abordan los primeros 17 meses desde el nacimiento.

\section{Procedimientos}

La intervención se realizó 3 veces por semana, durante 3 meses de programa de educación física, cada sesión duro 40 
minutos, que se dividieron en 5 minutos de calentamiento 30 de parte medular con actividad física moderada a vigorosa y 5 minutos para relajación. Para garantizar la innovación pedagógica en la fase medular mediante el programa $\mathrm{CATCH}$ que orienta la participación de los actores en el proceso enseñanza a aprendizaje, hacia una autoeficacia positiva, en base a cuatro objetivos de $\mathrm{CATCH}$, al impartir las clases de educación física:

1.-Participar en AFMV al menos el 50\% del tiempo. (Planificando cada sesión, técnicas efectivas para administrar la sesión, instrucciones claras y sencillas, establecer señas de inicio y alto de actividades, mantener una supervisión activa).

2.-Brindar al participante muchas oportunidades de practicar sus habilidades. (Todos deben contar con un material, evitar largas filas, evitar eliminación y limitar competencia, utilizar juegos cortos, evitar enfocarse a los más destacados).

3.-Participar en actividades físicas divertidas. (Planificando actividades divertidas y seguras, adaptando actividades y dirigirlas a varios niveles de actividad, recompensando conductas apropiadas, enfatizando la cooperación, modelando entusiasmo por la actividad física, nunca usar la actividad física como castigo).

4.-Motivar a los estudiantes a realizar actividad física fuera de la escuela. (Recompensando a los niños que las practiquen, recordando constante mente el beneficio de la actividad física).

Se orientó por parte del profesor que los estudiantes participaran en actividad física moderada a vigorosa al menos de 50\% del tiempo de cada sesión se utilizó el instrumento System for Observing Fitness and Instruction Time (SOFIT) Sistema para Observar el Tiempo de Instrucción de Actividad Física por sus siglas en inglés (Mckenzie, 2002; Mckenzie y col., 1992). La metodología se realizó previa capacitación con observadores entrenados que seleccionaron al azar por sorteo sencillo a 4 estudiantes ( 2 hombres y 2 mujeres) basados en la lista del grupo usando el esquema de procedimientos en el manual SOFIT, fueron observados en secuencia rotatoria de 12 intervalos durante 20 segundos cada uno, repitiéndose las observaciones durante toda la clase de educación física, para llevar a cabo esta actividad se utilizó un audio que guio las evaluaciones escuchándose con un reproductor de audio. Para determinar la intensidad se usaron códigos para clasificar los niveles de actividad, los cuales permitieron estimar el gasto energético asociado con la actividad física, este procedimiento se clasifico en cinco códigos: 1) acostado, 2) sentado, 3) parado, 4) caminando, y 5) muy activo que corresponde a correr o una actividad con un gasto energético mayor. A partir de la cuantificación de estos códigos se determinó el índice de actividad física moderada a vigorosa (IAFMV) sumando porcentualmente los códigos 4) caminando y 5) muy activo del total del tiempo de la clase de educación física.

\section{Análisis estadístico}

El análisis estadístico se realizó con el Programa Estadístico para las Ciencias Sociales (SPSS), versión 23.0 para Windows (IBM Corporation, New York, USA), calculando los valores descriptivos de las variables y los porcentajes de cambio ( $\ddot{\mathrm{A}} \%)$ ([(Mediapost- Mediapre)/Mediapre $]$ x 100 (Vincent, 1999); Para verificar la normalidad de los grupos y homogeneidad de la varianza de los datos, se utilizó el test estadístico de Shapiro-Wilk debido a la cantidad menor de 30 evaluaciones en los participantes presentando un grado de significancia de P-Valor e» 0.05 . Tomando en cuenta la metodología utilizada en la investigación se estableció una prueba de hipótesis alterna y otra nula, para su comprobación se utilizó la prueba t Student para muestras relacionadas con la finalidad de calcular la igualdad de la varianza, determinando un nivel de ád» 0.05 , es decir un 5\% como porcentaje de error de la prueba estadística.

\section{Resultados}

Los resultados de la estadística inferencial mediante el análisis estadístico test $\mathrm{t}$ Student para muestras relacionadas, reportó diferencias significativas en la puntuación de motricidad gruesa $(p=0.00)$ y motricidad fina $(p=0.00)$, Gráfica 1 y 2 respectivamente. Los valores de porcentajes de cambio $(\ddot{\mathrm{A}} \%)$ del estudio para el inicial y final [ (Mediapost Mediapre)/Mediapre] x 100; fueron de 25.5 Ä\% para la motricidad gruesa y $11.3 \ddot{\mathrm{A}} \%$ para la motricidad fina.

\begin{tabular}{|c|c|c|}
\hline \multirow{2}{*}{ Variables } & \multicolumn{2}{|c|}{$\mathrm{M} \pm \mathrm{DE}$} \\
\hline & Pre & Post \\
\hline Coordinación corporal & $82.80 \pm 8.8$ & $104.6 \pm 5.0$ \\
\hline Locomoción & $76.20 \pm 14.1$ & $87.95 \pm 11.8$ \\
\hline Motricidad gruesa & $80.60 \pm 8.7$ & $102.20 \pm 7.6$ \\
\hline Motricidad fina & $78.10 \pm 5.1$ & $82.7 \pm 3.8$ \\
\hline Habilidad Perceptiva & $84.60 \pm 14.0$ & $92.35 \pm 14.0$ \\
\hline Motricidad fina total & $80.55 \pm 12.8$ & $89.45 \pm 13.3$ \\
\hline
\end{tabular}
Batelle (Newborg y col., 1996).

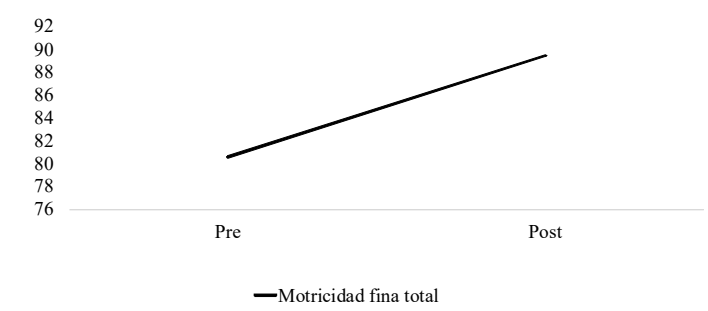

Gráfica 1. Cambios de la puntuación típica en la variable Motora Gruesa $(\mathrm{N}=20)$. Nota: Cálculo de la varianza de la variable Motricidad Gruesa mediante el test t student de muestras relacionadas $(\mathrm{p}=0.00)$, evaluado antes y después de la intervención con el inventario de desarrollo Battelle ( $\mathrm{n}=20)$. (Newborg y col., 1996).

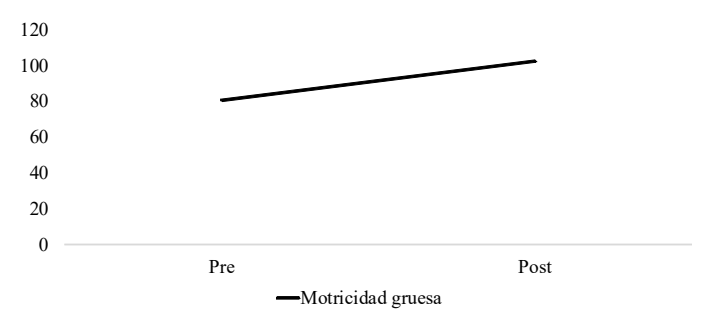

Gráfica 2. Cambios en la puntuación típica de la variable Motora Fina Total $(\mathrm{N}=20)$. Nota: Cálculo de la varianza de la variable Motricidad Fina Total mediante el test t student de muestras relacionadas $(\mathrm{p}=0.00)$, evaluado antes y después de la intervención con el inventario de desarrollo Battelle (n=20). (Newborg y col.,1996).

\section{Discusión}

El resultado principal del estudio fue, que tres meses de un programa de educación física adaptado al modelo pedagógico CATCH mejoran el desarrollo motor en niños de preescolar. Estos resultados son positivos, ya que una investigación en preescolares que utilizó el inventario de desarrollo 
Battelle, es consistente con nuestros resultados, demostrando que un adecuado desarrollo motor correlaciona con una adecuado desarrollo cognitivo y lenguaje, lo cual favorece el desarrollo integral de los niños (Campo Ternera, 2011).

Investigaciones han identificado que en clases de educación física la actividad física moderada vigorosa no está presente en más del 50\% del tiempo de la clase de educación física, (Hall y col., 2017, Flores Moreno, y col., 2017, Hall y col., 2018). El modelo pedagógico CATCH utilizado para esta investigación valoró una intensidad de la actividad física moderada a vigorosa fue $65 \%$ del tiempo total de la clase de educación física, intensidad que de acuerdo a los estándares establecidos por la NASPE (Asociación Nacional para el Deporte y la Educación Física, por sus siglas en inglés) coadyuvan a lograr la cantidad de actividad física recomendada para prescolares (Beets, y col., 2011). Investigaciones han asociado la práctica de actividad física moderada vigorosa con menor presencia de obesidad infantil (Ferrari, y col., 2015, Palencia y col., 2015) y se ha identificado que niños diagnosticados con sobrepeso y obesidad presentan una desigualdad de desarrollo motor comparado con niños con peso normal (Bucco y Zubiaur, 2015), un sin embargo un estudio implementados por dos años en preescolares utilizando el modelo CATCH, ha mostrado modestos resultados en disminuir el sobrepeso y obesidad (Shreela y col., 2019), lo en relación al desarrollo motor los beneficios se pueden observar en menor plazo tal es el caso de un estudio cuasi experimental reportó diferencias significativas en el desarrollo motor de niños de preescolar participantes en una intervención psicomotriz (Jiménez Díaz y Araya Vargas, 2009).

Se establece que por medio del movimiento, el desarrollo motriz en estudiantes de preescolar es favorecido con actividad física moderada vigorosa, a manera de juego brindando muchas oportunidades de participar en las accione motrices independientemente del género (Palma, Pereira, y Valentini, 2014), así como el uso de material didáctico que favorezca la igualdad de género (Moya-Mata, y col., 2019). Por lo que el profesorado juega un papel importante en la conducción directa de los contenidos en los programas educativos evitando estereotipos relacionados al género y dando la importancia de la igualdad de participación de los estudiantes en la clase de educación física (Alvariñas-Villaverde, y PazosGonzález, 2018).

El inventario de desarrollo Battelle, como instrumento es metodológicamente fácil de aplicar, económico y proporciona información relacionada a la coordinación motora, que puede ayudar al profesor de educación física a diagnosticar niños con dificultades de movimiento y diseñar actividades educativas enfocadas la condición motriz acorde a la edad (Sanz López, Guijarro Granados, y Sánchez Vázquez, 2007, Rizzoli-Córdoba, y col., 2013), así como realizar un abordaje multidisciplinario en torno a los niños con desarrollo motor bajo o deficiente (Barra Cabello, 2019).

En México el programa de educación física para preescolar presenta un enfoque didáctico enfocado en el desarrollo de la motricidad, integración de la corporeidad y la creatividad en la acción motriz (SEP, 2017), se identificado en por medio del movimiento que desarrollo motriz se favorece en estudiantes de prescolar con actividad física moderada vigorosa a manera de juego brindando muchas oportunidades de participar en las tareas motrices(Palma, Pereira, y Valentini, 2014), combinando estrategias didácticas como la expresión corporal e imaginación (Fernández Díez, y Arias García, 2015), incorporando fundamentos deportivos (Rodríguez Fernández, Pazos Couto, y Trigo Aza, 2016, Guillen Pereira, y col., 2018); Lo cual es fundamental considerarse dentro del formación del profesor de educación física y tener las competencias didácticas para la enseñanza en edades tempranas (Rodríguez Aceituno, y Hernández Pina, 2017) y tomar en cuenta factores que han identificado desigualdad en el desarrollo motor de los niños como el contexto educativo ya que se ha identificado que preescolares que estudian en el sector privado $(14,4 \%)$ presentan un porcentaje menor de desarrollo alterado que en el sector público (30,4\%) (Bedregal y col., 2016) de igual manera se ha identificado que en preescolares el nivel de desarrollo motor grueso para la edad, se encuentra en mejores niveles en población rural que en población urbana (Poblete et al., 2016), así como la condición socioeconómica, contexto familiar y la actividad física extraescolar son factores importantes a considerar en el desarrollo motor infantil (Herrera-Mora, 2019, Chiva-Bartoll, y Estevan, 2019).Como limitación se establece no contar con un grupo control ni tener previo conocimiento en los antecedentes de actividad física de los preescolares, en el futuro sería importante realizar un número mayor de estudios que clarifiquen situaciones causa efecto respecto a la motricidad de niños de edad preescolar, en diferentes ambientes pedagógicos y contextos sociales.

\section{Conclusiones}

A modo de conclusión, se destaca la importancia del desarrollo motriz fino y grueso obtenido en los preescolares, al participar durante tres meses en un programa de educación física con actividad física moderada a vigorosa adaptado al modelo pedagógico CATCH, que coadyuva desde la primera infancia en mejorar el desarrollo integral como lo recomienda la Organización Mundial de la Salud (OMS).

\section{Agradecimientos}

La investigación fue financiada por el Consejo Nacional de Ciencia y Tecnología CONACYT, del Padrón Nacional de Posgrados de Calidad PNPC. y aportando fondos a estudiante para estudiar en la Universidad Autónoma de Baja California, México y realizar una estancia de investigación en la Universidad de Huelva.

\section{Referencias}

Barra Cabello, L. (2019). Desafío diagnóstico e importancia del abordaje clínico del trastorno del desarrollo de la coordinación. Archivos argentinos de pediatría, 117(3), 199204. Recuperado de https://dx.doi.org/10.5546/aap.2019.199 Beets, M. W., Bornstein, D., Dowda, M., y Pate, R. R. (2011). Compliance with national guidelines for physical activity in U.S. preschoolers: measurement and interpretation. Pediatrics, 127(4), 658-664. Recuperado de doi:10.1542/peds.2010-2021

Bedregal, P., Hernández, V., Mingo, M. V., Castañón, C., Valenzuela, P., Moore, R., de la Cruz, R., y Castro, D. (2016). Desigualda- 
des en desarrollo infantil temprano entre prestadores públicos y privados de salud y factores asociados en la Región Metropolitana de Chile. Revista chilena de pediatría, 87(5), 351-358. Recuperado de https://dx.doi.org/10.1016/ j.rchipe.2016.02.008

Brooke, H. L., Corder, K., Atkin, A. J., y van Sluijs, E. M. (2014). A systematic literature review with meta-analyses of withinand between-day differences in objectively measured physical activity in school-aged children. Sports Medicine, 44(10),1427-38. Recuperado de doi: 10.1007/s40279-014-02155.

Bucco, L. y Zubiaur, M. (2015). Análisis del desarrollo motor en escolares brasileños con medidas corporales de obesidad y sobrepeso / Analysis of the Motor Development in Brazilian Schoolchildren with Corporal Measures of Obesity and Overweight. Revista Internacional de Medicina y Ciencias de la Actividad Física y el Deporte, 15 (59) 593-611. doi: http://dx.doi.org/10.15366/rimcafd2015.59.012

Campo Ternera, L. A. (2010). Importancia del desarrollo motor en relación con los procesos evolutivos del lenguaje y la cognición en niños de 3 a 7 años de la ciudad de Barranquilla (Colombia). Salud Uninorte, 26(1), 65-76. http:// w w w scielo.php?script $=$ sci arttext\&pid=S 0120 $55522010000100008 \& \operatorname{lng}=$ en\&tlng $=$.

Cigarroa, I., Sarqui, C., y Zapata-Lamana, R. (2016). Efectos del sedentarismo y obesidad en el desarrollo psicomotor en niños y niñas: Una revisión de la actualidad latinoamericana. Universidad y Salud, 18(1), 156-169. Recuperado de http://www.scielo.org.co/ scielophp?scaipt-sci arttext\&pid-S012471072016000100015\&ing-en\&thng-es

Chiva-Bartoll, O. y Estevan, I. (2019). El sexo, el contexto familiar y la actividad física extraescolar como factores asociados a la coordinación motriz en la niñez. Un estudio piloto. RICYDE. Revista internacional de ciencias del deporte, 56(15), 154170. Recuperado de https://doi.org/10.5232/ricyde2019.05603

Cuadri Fernández, J., Tornero Quiñones, I., Sierra Robles, Á., y Sáez Padilla, J. (2017). Revisión sistemática sobre los estudios de intervención de actividad física para el tratamiento de la obesidad (Systematic Review of Physical Activity Programs for the treatment of Obesity). Retos, 0(33), 261-266. Recuperado de https://recyt.fecyt.es/index.php/retos/article/ view $/ 52996$

Bermudez, M., Poblete, F., Pineda, A., Castro, N., y Inostroza, F. (2018). Nivel de desarrollo motor grueso en preescolares de México sin profesores de educación física. Revista Ciencias de la Actividad Física UCM, (1), 1-7. doi: http://doi.org/ 10.29035/rcaf.19.1.8

Fernández Díez, B., y Arias García, J. (2015). La Expresión Corporal como fuente de aprendizaje de nociones matemáticas espaciales en Educación Infantil (Corporal Expression as a means of learning spatial mathematical notions in Pre-School Education). Retos, 0(24), 158-164. Recuperado de https:// recyt.fecyt.es/index.php/retos/article/view/34550

Ferrari, G. L., Matsudo, V., Barreira, T. V., Tudor-Locke, C. Katzmarzyk, P. T, y Fisberg, M. (2016). Correlates of Moderate-to-Vigorous Physical Activity in Brazilian Children. Journal of Physical Activity and Health, 13(10):1132-1145. Recuperado de doi:10.1123/jpah.2015-0666

Flores Moreno, P., Salazar, C., Gómez Figueroa, J., Barreto Villa, Y., Valdovinos González, O., Vicente Rivera, J., y Del Río Valdivia, J. E. (2016). Medición del tiempo efectivo de la clase de educación física y su impacto en el gasto calórico en escolares de nivel primaria del municipio de Colima, México. Sportis. Scientific Journal of School Sport, Physical Education and Psychomotricity, 3(1), 34-49. Recuperado de https://doi.org/ 10.17979/sportis.2017.3.1.1766

Gil-Espinosa, F., Romance García, Á., y Nielsen Rodríguez, A. (2018). Juego y actividad física como indicadores de calidad en Educación Infantil (Games and physical activity as indicators of quality in Early Childhood Education). Retos, 0(34), 252-257. Recuperado de https://recyt.fecyt.es/ index.php/retos/article/view/60391

Guillen Pereira, L., Rojas Valladares, L., Formoso Mieres, A., Contreras Velázquez, L., y Estevez Pichs, M. (2018). Influencia de la Estimulación temprana en el desarrollo sensoriomotriz de niños de cuatro a seis años: una visión desde el Karate Do (Influence of early stimulation on the sensorimotor development of children aged four to six: a vision from Karate Do). Retos, 0(35), 147-155. Recuperado de https:// recyt.fecyt.es/index.php/retos/article/view/63104

Hall-López, J.A., Ochoa-Martínez, P.Y., Macías Castro, R., Zuñiga Burruel, R., y Sáenz-López Buñuel, P. (2018). Actividad física moderada a vigorosa en educación física y recreo en estudiantes de primaria y secundaria de la frontera MéxicoUSA. Sportis: Revista Técnico-Científica del Deporte Escolar, Educación Física y Psicomotricidad, 4(3), 426-442. Recuperado de https://doi.org/10.17979/sportis.2018.4.3.3175

Hall-López, J.A., Ochoa-Martínez, P.Y., Zuñiga Burruel, R., Macías Castro, R., y Sáenz-López Buñuel, P. (2017). Moderate-tovigorous physical activity during recess and physical education among Mexican elementary school students (Actividad física moderada a vigorosa durante el recreo y clase de educación física en niños mexicanos de escuela primaria). Retos, 0(31), 137-139. Recuperado de https://recyt.fecyt.es// index.php/retos/article/view/49640

Herrera-Mora, D. B., Munar-Torres, Y. E., Molina-Achury, N. J., y Robayo-Torres, A. L., (2019). Desarrollo infantil y condición socioeconómica. Artículo de revisión. Revista de la Facultad de Medicina, 67(1), 145-152. Recuperado de https:// dx.doi.org/10.15446/revfacmed.v67n1.66645

Jiménez Díaz, J., y Araya Vargas, G. (2009). Intervención motriz en el desarrollo motor, rendimiento académico y creatividad en preescolares. Pensar en movimiento, 7(1):11-22. Recuperado de http://www.redalyc.org/articulo.oa?id=442042959003

Jiménez Díaz, J., y Araya Vargas, G. (2010). Más minutos de educación física en preescolares favorecen el desarrollo motor. Pensar en movimiento, 8(1),1-8. Recuperado de doi: 10.15517/ PENSARMOV.V8I1.442

Kim, J. (2012). Are physical education-related state policies and schools' physical education requirement related to children's physical activity and obesity? Journal of School Health, 82(6):268-276. Recuperado de https://doi.org/10.1111/j.17461561.2012.00697.x

Langford, R., Bonell, C., Jones, H., Pouliou, T., Murphy, S., Waters, E., Komro, K., Gibbs, L., Magnus, D., y Campbell, R. (2015). The World Health Organization's Health Promoting Schools framework: a Cochrane systematic review and meta-analysis. BMC Public Health, 12;15:130. Recuperado de https:// doi.org/10.1186/s12889-015-1360-y

Lonsdale, C., Rosenkranz. R. R., Peralta, L. R., Bennie, A., Fahey, P., y Lubans, D.R. (2013). A systematic review and metaanalysis of interventions designed to increase moderate-tovigorous physical activity in school physical education lessons. Preventive Medicine, 56(2),152-61. Recuperado de doi:10.1016/j.ypmed.2012.12.004 
Luarte, R. C., Poblete, V. F., y Flores, R. C. (2014). Nivel de desarrollo motor grueso en preescolares sin intervención de profesores de educación física, Concepción, Chile. Ciencias De La Actividad Física UCM, 15(1), 7-16. Recuperado de http:/ /revistacaf.ucm.cl/article/view/36

Luna, P., y Poblete, F. (2011). Desarrollo motor en escolares sin intervención de profesionales de la educación física del nb1 del microcentro «amanecer» de la comuna de nacimiento (región del Biobío, Chile). Revista Horizonte Ciencias De La Actividad Física, 2(2), 25-35. Recuperado de http:// www.revistahorizonte.ulagos.cl/index.php/horizonte/article/ view/27

McKenzie, T.L., Strikmiller,P. K., Stone, E. J., Woods, S. E., Ehlinger, S.S ., Romero, K.A., y Budman, S. B. (1994). CATCH: Physical activity process evaluation in a multicenter trial. Health Education Quarterly, 2,S73-S89. Recuperado de https:// doi.org/10.1177/10901981940210S104

McKenzie, T., L. SOFIT. System for Observing Fitness Instruction Time. Overview and Training Manual. San Diego, CA: San Diego State University. 2002.

Mckenzie, T. L., Sallis, J. F., y Nader, P. R. (1992). Sofit-System for Observing Fitness Instruction Time. Journal of Teaching in Physical Education, 11(2),195-205.

Mollinedo Montaño, F., Esther, A., Piñón, E., Trejo-Ortiz, P. M., Araujo Espino, R., y Lugo Balderas, L. G. (2012). Relación del índice de masa corporal con el nivel de actividad física en preescolares. Revista Cubana de Enfermería, 28(2), 136-143. Recuperado de http://scielo.sld.cu/ scielo.php?script=sci_arttext\&pid=S086403192012000200009\&lng=es\&tlng=es.

Newborg, J., Stock, J, R., y Wnek, L. (1996). Inventario de Desarrollo Battelle, Madrid, Publicaciones de Psicología aplicada TEA.

Palencia, N. M. A., Martínez, M. S., Herráiz, M. M. G, Arribas, S. A., García, A. G, y Bueno, C. A. (2015). La actividad física vigorosa se relaciona con menor adiposidad y una condición física saludables en niños de 9-10 años. Revista Andaluza de Medicina del Deporte, 8(1), 38. Recuperado de https:/ /dx.doi.org/10.1016/j.ramd.2014.10.045

Palma, M., Pereira, B., \& Valentini, N. (2014). Guided play and free play in an enriched environment: Impact on motor development. Motriz: Revista de Educação Física, 20(2), 177-185. Recuperado de https://dx.doi.org/10.1590/S198065742014000200007

Poblete, F., Guerra, R., Toro, P., y Cruzat, E. (2016). Desarrollo motor grueso en escolares de zona urbana y rural. Revista Horizonte Ciencias De La Actividad Física, 7(1), 59-66. Recuperado de http://revistahorizonte.ulagos.cl/index.php/horizonte/article/view/73

Puri, K. S., Suresh, K. R., Gogtay, N. J., y Thatte, U. M. (2009). Declaration of Helsinki implications for stakeholders in research. Journal of Postgraduate Medicine, 55(2),131-134. Recuperado de doi: 10.4103/0022-3859.52846

Rizzoli-Córdoba, A., Schnaas-Arrieta, L., Liendo-Vallejos, S., Buenrostro-Márquez, G, Romo-Pardo, B., Carreón-García, J., Valadez-Correa, E., Scherer-Ibarra, P., López-Aranda, V., LiraGuerra, S., Robles-Anaya, R., Pizarro-Castellanos, M., Briones-Sandoval, A., Lia-Pirola, M., y Muñoz-Hernández, O. (2013). Validación de un instrumento para la detección oportuna de problemas de desarrollo en menores de 5 años en México. Boletín médico del Hospital Infantil de México, 70(3), 195-208. Recuperado de http://www.scielo.org.mx/ scielo.php?script=sci_arttext\&pid=S 1665 $11462013000300003 \& \operatorname{lng}=$ es\&tlng=es.
Rodriguez Aceituno, P., y Hernández Pina, F. (2017). Didáctica de la Motricidad en la formación de profesores de educación infantil (Didactics of Motricity in early childhood education teachers training). Retos, 0(34), 25-32. Recuperado de https:/ /recyt.fecyt.es/index.php/retos/article/view/58416

Rodríguez Fernández, J., Pazos Couto, J., y Trigo Aza, E. (2016). La gestión del deporte en clave educativa de Motricidad Humana (The management of the sport from an educational perspective in Human Kinetics). Retos, 0(30), 92-97. Recuperado de https://recyt.fecyt.es/index.php/retos/article/view/ 43909

Ruiz Heredia, C., Lara Sánchez, A., López Gallego, F., Cachón Zagalaz, J., y Valdivia Moral, P. (2018). Análisis del tiempo de clase en EF y propuestas para su optimización (Analysis of class time in physical education and proposals for optimization). Retos, 0(35), 126-129. Recuperado de https:// recyt.fecyt.es/index.php/retos/article/view/61880

Sanz López, Y., Guijarro Granados, T., y Sánchez Vázquez, V. Inventario de Desarrollo Battelle como instrumento de ayuda diagnóstica en el autismo. Revista de la Asociación Española de Neuropsiquiatría,27( 2 ): 31-45. Recuperado de h t t p : / / s c i e l o. i s c i i i. e s / scielo.php? script=sci_arttext\&pid=S021157352007000200004\&lng=es.

Schlechter, C. R., Rosenkranz, R. R., Fees, B. S., y Dzewaltowski, D. A. (2017). Preschool Daily Patterns of Physical Activity Driven by Location and Social Context. Journal of School Health ,87(3):194-199.Recuperado de https://doi.org/10.1111/josh.12486

Secretaria de Educación Pública (SEP) Aprendizajes Clave para la Educación Integral. Educación Física. Educación Básica. Plan y Programas de Estudio y sugerencias de evaluación. Primera edición2017, Ciudad de México. ISBN: 978-607-976444-9. Disponible también en la website: https:// www.aprendizajesclave.sep.gob.mx/

Shreela, V., Sharma, E., Ru-Jye, C., Courtney, B., Steven, K., Nancy, B., y Deanna, M. H. (2019). Impact of the Coordinated Approach to Child Health Early Childhood Program for Obesity Prevention among Preschool Children: The Texas Childhood Obesity Research Demonstration Study. Childhood Obesity, 15(1):1-13. Recuperado de doi: 10.1089/ chi.2018.0010.

Sims, J., Scarborough, P., y Foster, C. (2015). The Effectiveness of Interventions on Sustained Childhood Physical Activity: A Systematic Review and Meta-Analysis of Controlled Studies. PLoS One, 10(7), e0132935. Recuperado de doi:10.1371/ journal.pone. 0132935

Thomas, J. R., Nelson, J. K., y Silverman, S. J. Research Methods in Physical Activity (7th. Ed.). Human Kinetics. 2015. Champaign, Ilinois: Human Kinetics. ISBN: 9781450470445. PUBLICADO: http://www.humankinetics.com/products/allproducts/research-methods-in-physical-activity-7th-edition Vincent, W. J. (1999). Statistics in kinesiology (2nd Ed.). Champaign, Ilinois: Human Kinetics.

World health Organization WHO data revisited. (n.d.). Retrieved january 9, 2019, from the Global Strategy on Diet, Physical Activity and Health, Physical Activity and Young People, Recommended levels of physical activity for children aged 5-17 years; Recuperado de http://www.who.int/ dietphysicalactivity/factsheet_young_people/en/

World health Organization WHO data revisited. (n.d.). Retrieved january 17, 2019, from el Desarrollo de la primera infancia: un potente ecualizador; http://www.who.int/ maternal_child_adolescent/documents/ecd_final_m30/es 